\title{
Quark and Gluon iTMD parton distributions from HERA DIS data
}

\author{
Aleksandra Lelek* \\ DESY \\ E-mail: aleksandra.lelek@desy.de \\ Hannes Jung \\ $D E S Y$ \\ E-mail: hannes.jung@desy.de

\section{Francesco Hautmann} \\ University of Oxford \\ E-mail: Francesco.Hautmannephysics.ox.ac.uk
}

We describe a new approach to solve coupled quark and gluon DGLAP evolution equations with a Monte Carlo method. We show that this method is equivalent to other semi-analytical methods. We apply this method to extract quark and gluon parton densities (integrated over transverse momentum, iTMD), using the precision HERA DIS data.

38th International Conference on High Energy Physics 3-10 August 2016

Chicago, USA

${ }^{*}$ Speaker. 


\section{Motivation}

The quark and gluon collinear (integrated Transverse Momentum Dependent, iTMD) parton distribution functions are obtained using a Monte Carlo solution of the DGLAP evolution equations and we determine the starting distribution by fits to precision DIS data [1]. This method can be extended to determine TMDs.

In Ref. [2] the TMD gluon density was determined from DIS fits based on high-energy factorization [3] and CCFM evolution equations. This article is based on a different approach, in which we start from fully coupled quark and gluon DGLAP equations and solve these by a Monte Carlo method. The applied method is similar to the ones in Refs. [4, 5]. To perform a fit to DIS data we use an updated version of the program [6] within the xFitter open-source QCD platform [7].

\section{Introduction to the method}

The starting point of the discussion is the DGLAP evolution equation for momentum weighted parton density $x f\left(x, \mu^{2}\right)=\widetilde{f}\left(x, \mu^{2}\right)$

$$
\frac{d \widetilde{f}_{a}\left(x, \mu^{2}\right)}{d \ln \mu^{2}}=\sum_{b} \int_{x}^{1} \mathrm{dz} P_{a b}\left(\alpha_{s}\left(\mu^{2}\right), z\right) \widetilde{f}_{b}\left(\frac{x}{z}, \mu^{2}\right)
$$

where $a, b$ are quark ( $2 N_{f}$ flavours) or gluon, $x$ - longitudinal momentum fraction of the proton carried by a parton $a, z=x_{i} / x_{i-1}$ is the splitting variable and $\mu$ is the evolution mass scale. The splitting functions $P_{a b}$ have the following structure

$$
P_{a b}\left(\alpha_{s}\left(\mu^{2}\right), z\right)=D_{a b}\left(\alpha_{s}\left(\mu^{2}\right)\right) \delta(1-z)+K_{a b}\left(\alpha_{s}\left(\mu^{2}\right)\right) \frac{1}{(1-z)_{+}}+R_{a b}\left(\alpha_{s}\left(\mu^{2}\right), z\right)
$$

where $D_{a b}\left(\alpha_{s}\left(\mu^{2}\right)\right)=\delta_{a b} d_{a}\left(\alpha_{s}\left(\mu^{2}\right)\right), K_{a b}\left(\alpha_{s}\left(\mu^{2}\right)\right)=\delta_{a b} k_{a}\left(\alpha_{s}\left(\mu^{2}\right)\right)$ and $R_{a b}\left(\alpha_{s}\left(\mu^{2}\right), z\right)$ contains logarithmic terms in $\ln (1-z)$ and has no power divergences $(1-z)^{-n}$ for $z \rightarrow 1$.

Defining the Sudakov form factor as

$$
\Delta_{a}\left(\mu^{2}\right)=\exp \left(-\int_{\ln \mu_{0}^{2}}^{\ln \mu^{2}} d\left(\ln \mu^{\prime 2}\right) \sum_{b} \int_{0}^{1} d z z P_{b a}^{R}\left(\alpha_{s}\left(\mu^{\prime 2}\right), z\right)\right)
$$

and using momentum sum rule eq.(2.1) can be rewritten

$$
\frac{d \widetilde{f}_{a}\left(x, \mu^{2}\right)}{d \ln \mu^{2}}=\sum_{b} \int_{x}^{1} d z P_{a b}^{R}\left(\alpha_{s}\left(\mu^{2}\right), z\right) \widetilde{f}_{b}\left(\frac{x}{z}, \mu^{2}\right)+\widetilde{f}_{a}\left(x, \mu^{2}\right) \frac{1}{\Delta_{a}\left(\mu^{2}\right)} \frac{d \Delta_{a}\left(\mu^{2}\right)}{d \ln \mu^{2}} .
$$

where $P_{a b}^{R}\left(\alpha_{s}\left(\mu^{2}\right), z\right)=R_{a b}\left(\alpha_{s}\left(\mu^{2}\right), z\right)+K_{a b}\left(\alpha_{s}\left(\mu^{2}\right)\right) \frac{1}{1-z}$-real part of the splitting function.

This equation has an iterative solution which can be solved by a Monte Carlo method.

\section{3. iTMD evolution using MC method}

Some of the splitting functions are divergent for $z \rightarrow 1$. To avoid divergences, a cut off must be introduced and the upper limit in the integral over $z$ in eq. (2.4) as well as in the Sudakov form 
factor eq.(2.3) is put to $z_{\max }$ instead of 1 . It can be shown that the net effect of the skipped terms $\int_{z \max }^{1}$ is of order $\mathscr{O}\left(1-z_{\max }\right)$. There are different choices of $z_{\max }$ possible: $z_{\max }$ can be fixed or can change dynamically with the scale, for example like in angular ordering: $z_{\max }=1-\left(Q_{0} / Q\right)$. In this paper we present results for fixed $z_{\max }$.

In the Fig. 1 we show results for integrated distributions for gluons and down-quarks coming from the MC solution and from QCDNUM [8]. The initial distributions are taken from QCDNUM. We obtain a very good agreement between these two methods as soon as $z_{\max }$ is large enough. The Monte Carlo method provides an unbiased solution of the evolution equations and yields results fully consistent with those obtained from semi-analytical methods.
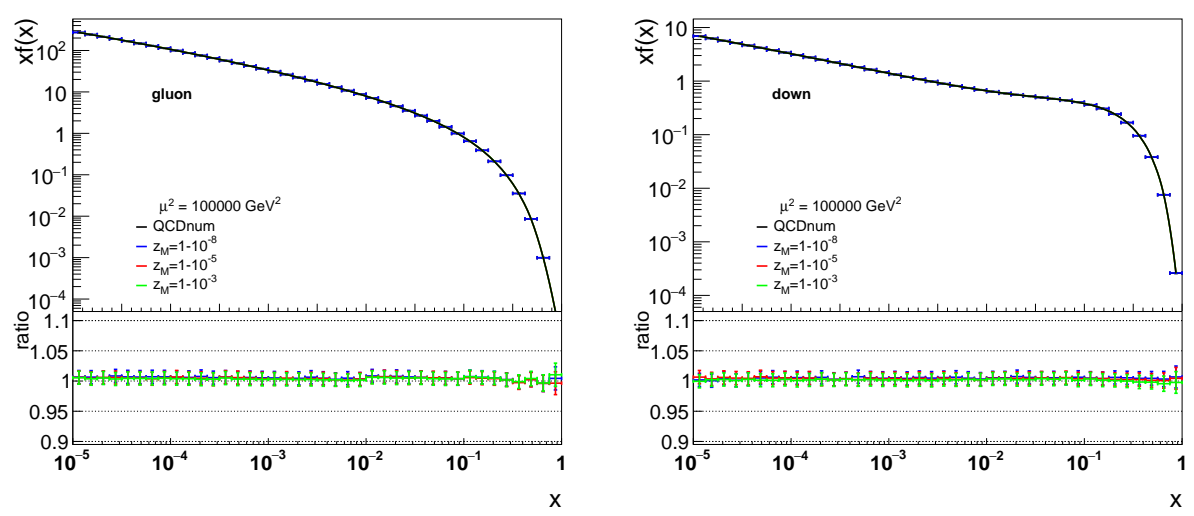

Figure 1: $x f(x, t)$ for gluon (left) and down quarks (right) as a function of $x$ after evolution up to $100000 \mathrm{GeV}^{2}$ compared to QCDNUM.

\section{4. iTMDs from a fit to HERA DIS data with xFitter}

As a consistency check, we performed the fit of iTMDs to $F_{2}$. This is also a check of the flavour decomposition with a MC method. In the past a gluon TMD pdf was fitted within xFitter [7] to $\sigma_{\text {red }}$ from H1/Zeus data for $Q^{2}>3.5 \mathrm{GeV}^{2}$ and $x<0.01$ [2]. Now we present the first fit of full (gluon, valence and sea) iTMDs to HERA H1 and Zeus data. Results are shown in the Fig. 2. The fit works reasonably well for $3.5<Q^{2}<30000 \mathrm{GeV}^{2}$ and $x>4 \cdot 10^{-5}\left(\chi^{2} /\right.$ ndf $\left.\approx 1\right)$.

\section{Summary}

A new approach to solve the coupled gluon and quark DGLAP evolution equation with a MC method was presented. Results coming from the MC solution reproduce semi-analytical results (QCDNUM). Parton distributions over the full range in $x$ and $Q^{2}$ are obtained from a Monte Carlo method to solve the evolution equation which is implemented in $x F i t t e r$. New results of fitting iTMD pdfs to $F_{2}$ with $\mathrm{xF}$ itter give $\chi^{2} / n d f \approx 1$ for $Q^{2}>3.5 \mathrm{GeV}^{2}$ for all $x$. 

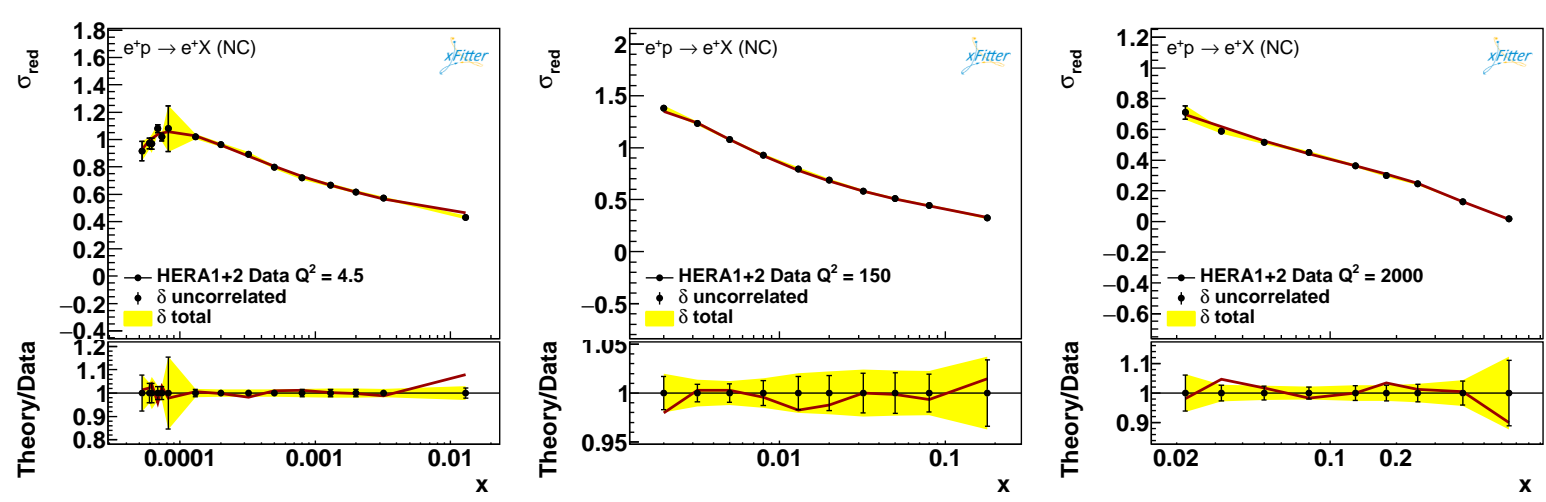

Figure 2: The reduced cross section $\sigma_{\text {red }}$ as measured at HERA compared to the LO order fits obtained with the parton branching method (red line) for different values of $Q^{2}$ obtained using xFitter.

\section{References}

[1] F. Aaron et al., JHEP 1001 (2010) 109.

[2] F. Hautmann and H. Jung, Nucl. Phys. B883 (2014) 1.

[3] S. Catani, M. Ciafaloni and F. Hautmann, Phys. Lett. B242 (1990) 97.

[4] H. Tanaka, PTEP 2015 (2015) 043B03.

[5] S. Jadach et al., Comput. Phys. Commun. 181 (2010) 393.

[6] F. Hautmann, H. Jung and S. Taheri Monfared, Eur. Phys. J. C 74 (2014) 3082.

[7] S. Alekhin et al., Eur. Phys. J. C 75 (2015) 304.

[8] M. Botje QCDNUM Comput. Phys. Commun. 182 (2011) 490 